\title{
8. Die Perspektive der Akteur*innen
}

Nachdem nun auf der Textebene herausgearbeitet wurde, auf welche Art und Weise die jeweiligen Projekte ihre queere Gemeinschaft diskursiv herstellen, geht es in diesem Abschnitt um die Frage, warum die einzelnen Aktivist"innen Ja sagen zu diesen queeren Gemeinschaften. Im Zentrum steht die Frage, welche Gründe die Aktivist"innen vor dem Hintergrund der projektspezifischen Bedingungs- und Bedeutungsanordnungen und des widersprüchlichen Verhältnisses von Identität und dem Politischen haben, sich für den politischen Aktivismus im Namen des jeweils projektspezifischen Wir zu entscheiden. Nachdem dieses projektspezifische Wir auf die eine oder andere Weise um den Begriff "queer « herum angeordnet ist, wurde nach dem Verhältnis der Aktivist"innen $\mathrm{zu}$ »queer«, insbesondere dem »queer«, das die jeweiligen Projekte zusammenhält, gefragt. Es ging aber auch generell darum, herauszuarbeiten, welche schließenden, aber auch Möglichkeiten eröffnenden Bedeutungen die Aktivist*innen in den diskursiven Anordnungen der Projekte erkennen und welche für sie relevant sind. Ausgangspunkt dieser Analyse ist auch hier der oft schwierige Versuch queerer Politiken, offenzuhalten, »wer sie sind «. ${ }^{1}$ Die Coming-out-Diskurse verweisen bereits darauf, dass im Namen eines individuellen Wohlbefindens ein Ja zur Identität trotz grundsätzlich queerer Haltung notwendig zu sein scheint. Auch die Philosophin Seyla Benhabib betont, dass auf die Frage nach der Rolle von Identität in sozialen Bewegungen aus der »Perspektive der ersten Person« eine andere Antwort zu erwarten sei: »Die Perspektive des gesellschaftlichen Beobachters dagegen darf nicht nur die historischen und soziologischen Kontingenzen dieser Bewegungen erklären, sondern muß auch aufzeigen, wie und warum Parteinahme und Kampf für Mitglieder dieser Bewegungen einleuchtend und erstrebenswert sind. Es muß also eine Ebene der Erläuterung von Handlungsmotiven geben. $\aleph^{2}$

Die Aufarbeitung queerer Diskurse, in denen die Projekte aushandeln, »wer sie sein wollen «, wird in diesem letzten Abschnitt um die Perspektive aus der ersten Person ergänzt. Allerdings fällt dieser Teil weniger umfangreich aus als der diskursanalytische

\footnotetext{
S. Hark: Wer wir sind und wie wir tun, S. 34.

S. Benhabib: Kulturelle Vielfalt und demokratische Gleichheit, S. 25.
} 
Teil. Sowohl die Fragestellung als auch der Gegenstand sind, wie bereits erläutert wurde, nur vor dem Hintergrund einer diskurstheoretischen Perspektive sinnvoll. Eine subjektwissenschaftliche ${ }^{3}$ Perspektive ist diskurstheoretisch nicht unbedingt naheliegend. Daher ist die Analyse von queeren Diskursen der zentrale Teil zur Beantwortung der Fragestellung der Arbeit. Dieser zweite, kleinere Teil ist hingegen ein Versuch, die diskursanalytische Perspektive um eine subjekttheoretische zu ergänzen. In der Subjektivierungsforschung gibt es mittlerweile einige Arbeiten, die auf Basis von Interviews die Perspektive der ersten Person einbinden, indem beispielsweise die »Selbsttechniken« der Subjekte herausgearbeitet werden. ${ }^{4}$ Diese Subjektivierungsforschungen bleiben allerdings meistens im Rahmen der Foucault'schen Diskurstheorie. Im Vergleich zu ihnen geht es in der Kritischen Psychologie weniger darum zu fragen, welche »Selbsttechniken« angewendet werden, sondern der Fokus liegt auf der Frage nach dem Warum. Mit diesem Fokus auf der Begründungsebene versucht die Kritische Psychologie, affektive, biographisch gewordene, aber auch situative Gründe der Akteur*innen, sich auf eine bestimmte Art und Weise in ein Verhältnis zu den diskursiven Anrufungen zu setzen, zu erfassen. Es geht in dem vorliegenden Teil nun darum, herauszuarbeiten, in welches Verhältnis die Aktivist*innen sich selbst zu den diskursiven Anordnungen ihrer Projekte stellen und welche Rolle dabei auf Schließung abzielende Identitäten spielen. Diese Perspektive soll einen Blick auf weitere Widersprüche eröffnen, die sich jenseits der rein diskursiven Anordnung ergeben. Was bedeutet es für die einzelnen Aktivist*innen, Ja zu sagen zu einem Wir, das versucht ist, sich nicht derart um (eindeutige) Identitäten herum anzuordnen? Welches queere Wir wollen die Aktivist"innen, welches nicht, und warum? Was haben die einzelnen Aktivist*innen zu gewinnen, was zu verlieren, wenn sie ein bestimmtes kollektives Wir annehmen? In welchem Verhältnis stehen die Begründungen der Aktivist"innen zu den diskursiven Aushandlungen eines queeren Wir? Um sich diesen Fragen zu nähern, wurde auf die Bedingungs-BedeutungsBegründungsanalyse (BBB) aus der Kritischen Psychologie und die Erhebungs- sowie Auswertungsmethode des problemzentrierten Interviews (PZI) von Andreas Witzel zurückgegriffen.

\subsection{Die Kritische Psychologie: Bedingungs-Bedeutungs-Begründungsanalyse}

Die Bedingungs-Bedeutungs-Begründungsstrukturen sind ein begriffliches Instrumentarium aus der Kritischen Psychologie, die 1969 vor allem an der Freien Universität Berlin ihre Anfänge nahm. Als prominenteste Vertreter*innen und Begründer*innen der Kritischen Psychologie gelten Klaus Holzkamp und Ute Osterkamp. Später wurden

3 Die Kritische Psychologie bezeichnet sich als Subjektwissenschaft.

4 Vgl. Lisa Pfahl-Lena Schürmann-Boris Taue: „Das Fleisch der Diskurse. Zur Verbindung von Biographie- und Diskursforschung in der wissenssoziologischen Subjektivierungsanalyse am Beispiel der Behindertenpädagogik«, in: Susann Fegter-Fabian Kessl-Antje Langer et al. (Hg.), Erziehungswissenschaftliche Diskursforschung. Empirische Analysen zu Bildungs- und Erziehungsverhältnissen, Wiesbaden: Springer VS 2015, S. 89-106. 
die Ideen und Konzepte vor allem durch Morus Markard, der den letzten Lehrstuhl für Kritische Psychologie in Berlin innehatte, sowie von Ole Dreier im englischsprachigen Raum verbreitet. ${ }^{5}$ Als bekannteste feministische Vertreterin, die der Kritischen Psychologie nahesteht, gilt die marxistische Feministin Frigga Haug. ${ }^{6}$ Aber auch Gerlinde Aumann hat die kritische psychologische Analyse von Geschlechterverhältnissen vorangetrieben. ${ }^{7}$ Die Kritische Psychologie ist im Zuge der 1968er-Student*innenproteste aus einer Kritik an der immer experimenteller und vermehrt quantitativ arbeitenden Psychologie heraus entstanden: Eine experimentell und quantitativ orientierte Psychologie könne das menschliche Leben und Erleben nicht als "gesamtgesellschaftlich vermitteltes « - so die Terminologie der Kritischen Psychologie erfassen. In der Anordnung experimenteller Studiendesigns, aber auch in den der Experimentellen Psychologie zugrundeliegenden Terminologien würde ein »abstraktisoliertes « Individuum - also ein Mensch, der nicht von Anfang an und grundlegend von gesellschaftlichen Verhältnissen durchdrungen ist - gedacht. Entsprechend könne und wolle die Psychologie gesellschaftliche Ungleichheitsverhältnisse (insbesondere Klassenverhältnisse) nicht mitdenken, geschweige denn hinterfragen. Stattdessen würden gesellschaftliche Widersprüche in das Individuum hinein verlagert. Die Notwendigkeit von Veränderungen werde so nur im einzelnen Menschen, nicht aber in den gesellschaftlichen Verhältnissen verortet. ${ }^{8}$

Die Kritische Psychologie, die als eine marxistische Subjektwissenschaft gilt, hatte demgegenüber das Ziel, ein begriffliches Analyseinstrumentarium zu entwickeln, das es möglich macht, das menschliche Erleben und Verhalten vor dem Hintergrund struktureller Ungleichheitsverhältnisse zu analysieren. Während beispielsweise Frigga Haug schon immer auch Geschlechterverhältnisse thematisierte, waren in der klassischen Kritischen Psychologie, wie sie in der "Grundlegung der Psychologie« von Klaus Holzkamp verankert ist, zunächst einmal nur Klassenverhältnisse zentral. Aktuell werden die in der »Grundlegung der Psychologie« entwickelten begrifflichen Instrumentarien für die Analyse von Geschlechterverhältnissen ambivalent bewertet: Auf der einen Seite liegt dem Denken der »Grundlegung der Psychologie« in Bezug auf Geschlechterund Familienverhältnisse ein biologistisches und heteronormatives Denken zugrunde. Auf der anderen Seite wird das begriffliche Instrumentarium für die Analyse von subjektivem Erleben vor dem Hintergrund des Klassenwiderspruchs als fruchtbar für die Analyse anderer Ungleichheitsverhältnisse - so auch Geschlechterverhältnisse - gesehen. ${ }^{9}$ Spätestens seitdem feministische Interventionen darauf verwiesen haben, dass S. 4-23; Morus Markard: Einführung in die Kritische Psychologie, Hamburg: Argument Verlag 2009.

$6 \quad$ Frigga Haug war allerdings in vielen Fragen uneinig mit der klassischen Holzkamp-Schule. Vgl. Frigga Haug: »Lernen lehren und Lehren lernen«, in: Forum Kritische Psychologie (2013), S. 34-62.

7 Vgl. Gerlinde Aumann: Kritische Psychologie und Psychoanalyse. Historisch-subjektwissenschaftliche Analyse zum Ceschlechterverhältnis, Hamburg: Argument Verlag 2003.

8 M. Markard: Einführung in die Kritische Psychologie.

9 Vgl. Anna Sieben-Fiona Kalkstein: »Kritische Psychologie und queer-feministische Perspektiven: Möglichkeiten und Grenzen einer Wiederaneignung der Arbeiten von Klaus Holzkamp und Ute Holzkamp-Osterkamp«, in: Journal für Psychologie 23 (2015), S. 233-258. 
Frauen durch ihre unbezahlten Sorgetätigkeiten die Reproduktion der Produktionsbedingungen sichern, ist deutlich geworden, dass Klassen- und Geschlechterverhältnisse untrennbar miteinander verknüpft sind und sich wechselseitig konstituieren. Entsprechend kommt eine Analyse des psychischen Erlebens vor dem Hintergrund von sozialen Ungleichheitsverhältnissen, wie sie die Kritische Psychologie vorschlägt, nicht mehr umhin, Geschlechterverhältnisse mitzudenken. ${ }^{10}$ Die begrifflichen Instrumentarien der Kritischen Psychologie sind für die Analyse von Geschlechterverhältnissen gerade deswegen von (Mehr-)Wert, weil sie den Anspruch haben, das psychische Erleben in seinem konstitutiven Wechselverhältnis mit gesellschaftlichen Strukturen und Bedeutungsanordnungen zu erfassen. Jäger, dessen Kritische Diskursanalyse in den ersten Auflagen noch einen expliziten Bezug zur Kritischen Psychologie aufweist, verortet die Relevanz der Kritischen Psychologie in der Möglichkeit, das Verhältnis zwischen Diskurs und Subjekt näher zu bestimmen: „Das (tätige) Subjekt, auch wenn es als einzelnes den Diskurs bzw. die Aussagen, die den Diskurs ausmachen, natürlich nicht >macht<, ist aber doch immer mit seinen Äußerungen, seinem Wissen und seinen Fähigkeiten daran beteiligt und muss sich den Wirkungen des Diskurses ja nicht unkritisch und unbewusst aussetzen. ${ }^{11}$ Um das Verhältnis zwischen Subjekt, Diskurs und Strukturen empirisch erfassen zu können, ist in den Jahren nach den Arbeiten von Holzkamp die Bedingungs-Bedeutungs-Begründungsanalyse als ein methodisches Analyseraster weiterentwickelt worden. ${ }^{12}$ Die Begriffe selbst haben ihren Ursprung in der »Grundlegung der Psychologie«, die Klaus Holzkamp 1983 veröffentlicht hat. ${ }^{13}$ Sie stellen, in Abgrenzung zur Experimentellen Psychologie, Versuche dar, das MenschWelt-Verhältnis vor dem Hintergrund sozialer Ungleichheiten denkbar und analysierbar zu machen. Was versteht nun aber die Kritische Psychologie unter Bedingungen, Bedeutungen und Begründungen ${ }^{14}$

Während die Bedingungs- und die Bedeutungsebene eher die das Erleben des Einzelnen von Anfang an durchdringenden gesellschaftlichen Verhältnisse fassbar

10 Lisa Malich-Tanja Vogler: «Kritische Psychologie mit kleinem q«, in: Journal für Psychologie 26 (2018), S. 160-183, hier S. 166f.

11 R. Diaz-Bone: Kritische Diskursanalyse: Zur Ausarbeitung einer problembezogenen Diskursanalyse im Anschluss an Foucault. Siegfried Jäger im Cespräch mit Rainer Diaz-Bone.

12 Vgl. Morus Markard: »Kritische Psychologie: Forschung vom Standpunkt des Subjekts«, in: Cünter Mey-Katja Mruck (Hg.), Handbuch Qualitative Forschung in der Psychologie, Wiesbaden: Springer Verlag 2010, S. 166-181.

13 Klaus Holzkamp: Grundlegung der Psychologie, Frankfurt a.M.: Campus Verlag 1983.

14 Zunächst einmal ist zu betonen, dass Hozkamps stark marxistisch geprägtes Analyseinstrumentarium durch eine diskurstheoretisch geprägte Brille und so auch zum Teil mit Holzkamp gegen Holzkamp gelesen wird. Auch wenn dieser sich vor allem in seinen späteren Werken mit Foucault auseinandergesetzt hat und sich affirmativ auf seine Arbeiten, aber auch sein Machtverständnis bezieht, hat dieser niemals von Subjektivierung, Selbstverhältnissen oder der Möglichkeit, sich nicht derart zu unterwerfen, gesprochen. Vielmehr umfasst das begriffliche Instrumentarium der Kritischen Psychologie nach Holzkamp eher Begriffe wie »Handlungsfähigkeit«, »gesellschaftliche Vermitteltheit «, »sich verhalten zu « und »restriktive Handlungsfähigkeit«. Cemeinsam ist dem Denken der Kritischen Psychologie und jenem der Diskurstheorie, dass Menschen niemals als den gesellschaftlichen - diskursiven wie materiellen - Verhältnissen, die sie durchdringen, vorgängig gesehen werden. 
machen, stellt die Begründungsebene die subjektive Komponente dieses Analyserasters dar. Dabei überschneiden und bedingen sich die drei Ebenen und sind nur analytisch voneinander trennbar. Unter Bedingungen versteht die Kritische Psychologie in Anlehnung an marxistische Perspektiven vor allem materielle strukturelle Bedingungen, wie das für unsere Gesellschaft grundlegende Verhältnis von Kapital und Arbeit. ${ }^{15}$ Bedeutungsstrukturen können wiederum als Diskurse verstanden werden, die die Denk- und Handlungsmöglichkeiten der Menschen in der Welt rahmen und gesellschaftliche Bedingungen vermitteln: ${ }^{16}$ »[1]hr bewusstes >Verhalten etc. dazu ist immer eine subjektive Realisierung der aufgewiesenen gesellschaftlichen Bedeutungsstrukturen, insbesondere in ihrer >symbolischen Repräsentanz durch die 'gnostischen Strukturen gesellschaftlicher Denk- und Sprachformen. ${ }^{17}$ Während die Bedingungs- und Bedeutungsebenen (auch) gesellschaftstheoretisch erfasst werden können, stellt die Begründungsebene ausschließlich eine subjektive Perspektive dar. Vor dem Hintergrund, dass Bedingungs- und Bedeutungsanordnungen die einzelnen Menschen nicht determinieren, sondern einen Rahmen vorgeben, in dem sie an, mit und gegen bestimmte Arten, zum Subjekt zu werden, arbeiten können, wird auf der Begründungsebene die Frage nach dem Warum gestellt. Während gesellschaftliche Diskurse Subjektivierungs-/Handlungsmöglichkeiten bereitstellen, kann mit ihnen noch nicht erklärt werden, warum manche Subjekte sich den diskursiven Möglichkeiten, zum Subjekt zu werden, unterwerfen und andere nicht oder nicht derart. ${ }^{18} \mathrm{Genau}$ diesem Aspekt will Holzkamp mit dem Konzept der subjektiven Handlungsgründe Rechnung tragen: »Menschliche Handlungen/Befindlichkeiten sind also weder bloß unmittelbar äußerlich >bedingt<, noch sind sie Resultat bloß >subjektiver Bedeutungsstiftungen o.ä., sondern sie sind in den Lebensbedingungen >begründet « ${ }^{19}$ Es geht in der Kritisch-Psychologischen Terminologie darum, zu fragen, warum und auf welche bestimmte Art und Weise sich die Menschen zu den Möglichkeiten, die ihnen bestimmte Bedingungs-Bedeutungskonstellationen eröffnen, selbst ins Verhältnis setzen. ${ }^{20}$ Es ist von Interesse, was sie sich dabei zu gewinnen erhoffen und was sie dabei riskieren. Um diese Frage nach dem Warum stellen zu können, wird zunächst auf einer subjektiven Ebene danach gefragt, welche gesellschaftlichen Bedingungsund Bedeutungskonstellationen sichtbar und relevant sind. ${ }^{21}$ Diese Sichtbarkeit und

Ergänzt um marxistisch-feministische Erkenntnisse muss hier auch das vergeschlechtlichte Verhältnis von Arbeit und Kapital mitgedacht werden. L. Malich-T. Vogler: »Kritische Psychologie mit kleinem q«, S. 163-169.

16 Ebd., S. 169-175.

17 K. Holzkamp: Crundlegung der Psychologie, S. $347 f$.

18 Vgl. S. Hall: Ideologie, Identität, Repräsentation, S. 168.

19 K. Holzkamp: Crundlegung der Psychologie, S. 348.

20 Dabei werden die einzelnen Menschen, deren subjektives Erleben herausgearbeitet wird, auch in der Kritischen Psychologie niemals als den gesellschaftlichen Bedingungs- und Bedeutungsanordnungen, zu denen sie ein bestimmtes Selbstverhältnis entwickeln, vorgängig verstanden. Klaus Holzkamp: »Gesellschaftliche Widersprüche und individuelle Handlungsfähigkeit am Beispiel der Sozialarbeit«, in: Ulrike Eichinger-Klaus Weber (Hg.), Soziale Arbeit, Hamburg: Argument 2012, S. 16-40, hier S. 27.

21 In manchen Arbeiten werden Bedingungs-Bedeutungsanordnungen auch auf einer gesamtgesellschaftlichen Ebene erfasst. Hier findet ausschließlich eine Herausarbeitung der subjektiv erfah- 
Relevanz, aber auch die konkrete Begründungsebene hängt zum einen mit der spezifischen Lebenslage zusammen, in der die Subjekte sich befinden, zum anderen aber auch mit dem, was sie aus ihren biographischen Erfahrungen heraus geworden sind. ${ }^{22}$ Es geht darum zu verstehen, wie sie als die Subjekte, die sie geworden sind und die sie ständig werden, die Bedingungs-Bedeutungsanordnungen erleben und welche sie warum für mehr oder weniger relevant erachten. Zu verstehen, warum Menschen sich auf eine bestimmte Art und Weise zu den Bedingungs-Bedeutungsanordnungen, die sie umgeben, verhalten, ist in der Kritischen Psychologie auch deswegen relevant, weil aus einer subjektiven Perspektive heraus verständlich wird, warum Menschen immer wieder Ja sagen zu gesellschaftlichen Verhältnissen, die Ungleichheiten produzieren. Es geht darum zu verstehen, warum sich Menschen bestimmten Bedingungen der Subjektwerdung unterwerfen oder versuchen, sich nicht derart $\mathrm{zu}$ unterwerfen. ${ }^{23}$ Indem mit Hilfe der BBB herausgearbeitet wird, was die Einzelnen zu gewinnen oder $\mathrm{zu}$ verlieren haben beziehungsweise warum sie ein Interesse haben, an, mit oder gegen bestimmte Ungleichheitsverhältnisse zu arbeiten, kann eine weitere Perspektive auf die Möglichkeiten und Grenzen der Herstellung eines kollektiven Wir gelegt werden. Je nachdem, welche Rolle ein solches Wir und die Möglichkeiten, die durch ebendieses eröffnet werden, für die Akteur"innen spielen und welche Bedürfnisse die Akteur"innen, die in die Bewegung eintreten, darüber hinaus haben, kann auch die Antwort auf die Frage nach der Rolle von Identitäten für dieses Wir aus der Perspektive des Subjekts noch einmal anders ausfallen.

\subsection{Das problemzentrierte Interview: Erhebung und Auswertung}

Um dieser Frage nachzugehen, wurden problemzentrierte Interviews (PZI) mit Aktivist*innen aus den im Zentrum der vorliegenden Untersuchungen stehenden queeren Projekten durchgeführt. Nachdem die handlungstheoretischen Grundannahmen des PZI schwer mit einem diskurstheoretischen Denken vereinbar sind, ${ }^{24}$ stellt das PZI in

renen Bedingungs- und Bedeutungsanordnungen statt. Aspekte gesellschaftlicher BedingungsBedeutungsanordnungen wurden auf theoretischer und bewegungsgeschichtlicher sowie diskursiver Ebene bereits in den vorherigen Kapiteln aufgearbeitet.

22 K. Holzkamp: Grundlegung der Psychologie, S. 353.

23 Von einem solchen Verständnis ausgehend, versucht die Kritisch-Psychologische Forschung, die sich zumeist als partizipatorische Forschung versteht, gemeinsam mit den »Beforschten «- die als Mitforscher*innen verstanden werden - alternative, weniger »restriktive« Handlungsmöglichkeiten zu eröffnen. L. Malich-T. Vogler: »Kritische Psychologie mit kleinem q«, S. $177 f$.

24 Das PZI geht davon aus, dass das Subjekt in Aushandlung von normativen und sozialen Strukturen und persönlicher Orientierung eigene Lebenszusammenhänge herstellt. Interviewte Personen werden entsprechend als Expert*innen ihrer Erfahrungen und Lebensumstände betrachtet, gerade weil sie die Akteur*innen ihrer Lebensverhältnisse sind. Damit geht das PZI im Unterschied zur Diskurstheorie zum einen von einem starken Akteur*innenbegriff aus, zum anderen wird den Akteur*innen etwas Eigenes - eine persönliche Orientierung-zugeschrieben. Witzel, Andreas: »Das problemzentrierte Interview«, in: Cerd ]üttemann (Hg.), Qualitative Forschung in der Psychologie: Crundfragen, Verfahrensweisen, Anwendungsfelder, Heidelberg: Asanger-Verlag 1989, S. 227-255. 
der vorliegenden Arbeit lediglich das Erhebungsinstrument dar, dass vor dem Hintergrund des handlungstheoretischen Modells der BBB eingesetzt wird. Das problemzentrierte Interview wurde unter anderem ausgewählt, weil es die Möglichkeit bietet, in der Erhebungsphase das Gespräch um einen Widerspruch beziehungsweise ein Problem herum anzuordnen. ${ }^{25}$ Das ermöglicht es zum einen, im Sinne der Fragestellung das widersprüchliche Verhältnis von queeren Politiken und Identitäten in den Blick zu nehmen, zum anderen ist ein Problem oder Handlungswiderspruch immer auch Ausgangspunkt der Bedingungs-Bedeutungs-Begründungsanalyse. Ein weiterer Aspekt, der für das problemzentrierte Interview spricht, ist der zugleich offene und theoriegeleitete Erhebungs- und Auswertungsprozess. Die Theoriegeleitetheit, die Witzel einem »naiv induktivistischen « Modell qualitativer Forschung entgegensetzt, ${ }^{26}$ ist gut in Einklang $\mathrm{zu}$ bringen mit dem begrifflichen Analyseinstrumentarium der BBB. Denn die Begrifflichkeiten der BBB sind, wie bereits ausgeführt wurde, auch aus einer Kritik an theoretisch wenig ausgearbeiteten und vermeintlich unvoreingenommenen Konstrukten der Experimentellen Psychologie heraus entwickelt worden. Das problemzentrierte Interview will aber trotz Theoriegeleitetheit nicht umgekehrt blind einem rein deduktiven Modell verfallen, das eine Offenheit gegenüber den Relevanzsetzungen der Subjekte verunmöglicht. Auch diese Haltung stimmt mit den Grundsätzen der Kritischen Psychologie überein, die etwa an den quantitativen Verfahren der Psychologie kritisiert, dass deren Messungen und Erhebungsmethoden - Fragebogen und Experiment - die subjektive Perspektive außen vor lassen. Witzel schlägt in Abgrenzung zu rein deduktiven oder induktiven Verfahren eine Methode vor, in der Offenheit und Theoriegeleitetheit sich abwechseln: »Theoretische Aussagen werden auf der Basis des vorhandenen Datenmaterials und des vorhandenen theoretischen Wissens formuliert. ${ }^{27}$ Ein Changieren zwischen Theoriegeleitetheit und Offenheit findet sich beim problemzentrierten Interview sowohl in der Erhebungs- als auch in der Auswertungsphase wieder.

Im Erhebungsprozess des problemzentrierten Interviews dient die Theorie zunächst als »heuristisch-analytischer Rahmen«, um »Frageideen « und Interviewleitfaden zu konzipieren. ${ }^{28}$ Der Erhebungsphase sind in der vorliegenden Untersuchung entsprechend auf verschiedenen Ebenen theoretische Vorannahmen, aber auch eine Offenheit für den Gegenstand vorausgegangen: Bereits das Problem, um das das Interview angeordnet ist, basiert auf einer Verknüpfung der Fragestellung mit dem handlungstheoretischen Konzept der BBB. Auch bei der Auswahl der Projekte, aus denen die Interviewpartner*innen stammen, ist zwischen Theoriegeleitetheit und Offenheit für den potentiellen Gegenstand changiert worden. ${ }^{29}$ Das der BBB zugrunde liegende Handlungsmodell hat sowohl die Erstellung des Leitfadens als auch die Gesprächsführung (mit-)strukturiert. Entsprechend wurden für den Interviewleitfaden Fragen und Themenfelder generiert, die es ermöglichten, nach den subjektiven

Andreas Witzel: »Das problemzentrierte Interview«, in: Forum Qualitative Sozialforschung 1 (2000), S. 463-475.

A. Witzel: Auswertung problemzentrierter Interviews. Grundlagen und Erfahrungen, S. 52.

Ebd.

Der genaue Auswahlprozess wird in Kapitel vier ausführlich beschrieben. 
Bedingungs-/Bedeutungs-/Begründungsaspekten der Aktivist"innen in Bezug auf ihre Entscheidung, sich in dem jeweiligen Projekt zu engagieren, zu fragen. In Anlehnung an die Offenheit des problemzentrierten Interviews wurde versucht, zum einen vor allem erzählgenerierende Fragen zu stellen und zum anderen dem Gesprächs- und Gedankenverlauf der Interviewpartner*innen zu folgen: „Das bedeutet, daß Kommunikationsstrategien zunächst das Offenheitsprinzip ermöglichen müssen, um beim Interviewpartner z.B. Gedankenschleifen, Korrekturen durch zunehmende Erinnerungen, weitere Beispiele und insgesamt seine Art und Weise der Entfaltung und Formulierung der in Frage stehende Thematik anzuregen. $\aleph^{30}$ Der Interviewleitfaden diente dabei als Unterstützung in dem Bemühen, das Interview um das Problem zu zentrieren. Die Leitfragen wurden in einem Probeinterview getestet. Zudem wurde er im Laufe der Erhebungsphasen weiterentwickelt und an die jeweiligen Interviewsituationen angepasst. Die Interviewpartner*innen wurden alle bereits bei der ersten Kontaktaufnahme über die dem Interview zugrunde liegende Fragestellung informiert. Die erste Kontaktaufnahme hat schon in der Konzeptionsphase des Dissertationsprojektes im Jahr 2015 stattgefunden. Die Interviews wurden dann zwischen Juni 2017 und Februar 2018 durchgeführt. Dabei hat es sich trotz anfänglicher Zusagen, dass ein Interview möglich sei, und trotz Unterstützung von Seiten der jeweiligen Projekte als schwierig erwiesen, Interviewpartner*innen zu finden. Drei Interviews waren letztendlich nur über mehrere Versuche der Kontaktaufnahme hinweg und aufgrund verschiedener persönlicher Kontakte möglich. ${ }^{31}$ Das liegt an der extrem hohen Interviewnachfrage, mit der die Einrichtungen konfrontiert sind, an den prekären Verhältnissen, in denen die teils ehrenamtliche, teils bezahlte Arbeit stattfindet, an der Tatsache, dass die Einrichtungen teilweise völlig überlastet sind, weil es nicht genügend Einrichtungen dieser Art gibt, aber auch an einer Skepsis, die der Aktivismus zum Teil gegenüber der »akademischen « Welt hat. Insgesamt wurde mit mindestens je einer Person aus jedem der fünf Projekte ein Interview durchgeführt. ${ }^{32}$ Die Interviews dauern zwischen einer halben Stunde und einer Stunde. Das Interview mit den Aktivist*innen der Milchjugend hat am Stand der Milchjugend auf dem Festival-Gelände der Pride in Zürich stattgefunden. ${ }^{33}$ Das Interview mit der Person aus der Villa ${ }^{34}$ und jener aus dem Jugendnetzwerk Lambda BB hat in einem Café stattgefunden und die Personen von TrIQ und LesMigraS wurden jeweils in den Räumen des Projektes interviewt. Die Interviewpartner*innen arbeiten teils ehrenamtlich, teils bezahlt, manche sowohl bezahlt als auch ehrenamtlich in den jeweiligen Projekten. Sie positionierten sich selbst

30 A. Witzel: Auswertung problemzentrierter Interviews. Grundlagen und Erfahrungen, S. 55.

31 An dieser Stelle möchte ich mich bei meinen Interviewpartner"innen, die sich die Zeit genommen haben, und bei allen, die mich auf der Suche nach Interviewpartner*innen unterstützt haben, bedanken.

32 Bei der Milchjugend wurden zwei Personen interviewt.

33 Die Entscheidung für diesen Ort ist gefallen, weil die Milchjugend im Cegensatz zu anderen Projekten, außer einem kleinen Büro, keine eigenen Räumlichkeiten hat, aber auch, weil mir dieser Ort die Möglichkeit geboten hat, die Milchjugend auf der Pride mitzuerleben.

34 Allerdings gab es - anlässlich eines Besuchs des Archivs - bereits ein Jahr vorher ein Treffen mit der interviewten Person in der Villa. 
als Inter*, Trans*, schwul, lesbisch, queer, manche mit Mehrfachdiskriminierungserfahrungen, andere nicht. Alle Interviewpartner*innen haben einen akademischen Abschluss oder studieren noch. Eine interviewte Person war zum Zeitpunkt des Interviews erst seit einem halben Jahr in dem Projekt aktiv, ${ }^{35}$ alle anderen schon längere Zeit. Zwei der interviewten Aktivist"innen waren zum Zeitpunkt des Interviews nicht mehr in der jeweiligen Einrichtung engagiert. In diesem Fall eröffnete sich insofern eine Möglichkeit der Kontrastierung, als die Interviewten nicht nur über ihre Gründe für ein Engagement gesprochen haben, sondern auch darüber, warum sie es beendet haben. Eine Zuordnung zu den jeweiligen Projekten findet unter ausdrücklicher Erlaubnis der interviewten Personen statt. Von der Erlaubnis Gebrauch gemacht wird nur dann, wenn es für das Verständnis des Sachverhalts von Bedeutung ist. Die Interviews wurden mit dem Einverständnis der Interviewpartner*innen aufgenommen und vollständig transkribiert. ${ }^{36}$ Im Anschluss an die Erhebungsphase wurde analog zu der von Witzel vorgeschlagenen Vorgangsweise ein »Postskriptum « erstellt, in dem erste für die Analyse vermeintlich wichtige Aspekte und Stimmungen des Gesprächs, aber auch Relevantes aus dem informellen Gespräch nach dem Interview festgehalten wurden. ${ }^{37}$

Die Auswertung der Interviews hat erst im Anschluss an die vollständige Auswertung des diskursanalytischen Materials stattgefunden. Auch sie ist an das Verfahren des problemzentrierten Interviews bei Witzel angelehnt, das wiederum vor allem auf die Grounded Theory zurückgeht. Die erste Auswertungsphase beginnt mit der »offenen Kodierung«. Auch hier wechseln sich die Prinzipien der Offenheit und Theoriegeleitetheit ab. Grundsätzlich werden beim »offenen Kodieren« die Interview-Transkripte Satz für Satz durchgearbeitet und mit Kodes versehen: »Die Resultate dieses Interpretationsprozesses bestehen zunächst in der Markierung des Textes mit Stichworten aus dem Leitfaden (theoriegeleitet) und aus Relevanzsetzungen der dem Interviewpartner entnommenen Begrifflichkeiten im Text selbst (Offenheitsprinzip). ${ }^{38}$ Entsprechend wurden die Interviews in der Phase des »offenen Kodierens", im Sinne der Offenheit, mit »in vivo«-Kodes versehen, die auf die »Relevanzsetzung « der Interviewpartner*innen verweisen. $\mathrm{Zu}$ dieser ersten Phase des »offenen Kodierens « gehört, ebenfalls im Sinne Witzels, eine »Falldarstellung«: »Sie [die Falldarstellung] ist eher deskriptiv und dient dazu, sich mit den wesentlichen Details des Einzelfalls vertraut zu machen. «9 Bereits in dieser ersten Phase des »offenen Kodierens« haben sich relevante Themen herauskristallisiert, um die herum die nächste Phase des Kodierens angeordnet wurde. $\mathrm{Zu}$ nächst einmal war der Frage nach der Art und Weise, wie die Aktivist*innen sich selbst zum »Queer« ihrer Projekte ins Verhältnis setzen, zentral. Dieser Themenkomplex ist in

Allerdings war diese Person vorher in einem ähnlichen Projekt in einer anderen Stadt aktiv.

Einigen Aktivist*innen wurde, wie im Vorgespräch vereinbart, die Ergebnisdarstellung vor der Veröffentlichung der Arbeit zugeschickt. Einige wenige direkte Interviewzitate wurden auf Wunsch der interviewten Personen minimal abgeändert, um beispielsweise die Anonymität zu gewährleisten oder das Zentrale einer Aussage, etwa durch eine Änderung der Satzzeichen, besser zu betonen.

A. Witzel: Auswertung problemzentrierter Interviews. Grundlagen und Erfahrungen, S. 57.

Ebd., S. 59.

Ebd., S. 60. 
der Erhebungsphase eigens abgefragt worden. Jenseits dessen, was spezifisch erfragt wurde, haben sich aus der offenen Interviewführung heraus weitere Aspekte, die relevant in Bezug auf die Entscheidung für oder gegen das Wir der Projekte sind, ergeben. Die offene Anordnung des Interviews hat aber auch gezeigt, dass die Aktivist"innen explizit Bezug nehmen auf die Aspekte, die in den diskursiven Anordnungen der Bewegungsmaterialien zentral sind. So spielen Themen wie Intersektionalität und Mehrfachdiskriminierung, Pride und Coming-out eine Rolle in der Entscheidung für und gegen die queeren Projekte. Neben diesen drei zentralen Themenkomplexen gibt es noch weitere subjektive Bedingungs-/Bedeutungs-/Begründunganordnungen, die mitunter sehr spezifisch sind und sich nicht einer übergeordneten Kategorie zuordnen lassen.

Die zweite Phase der Auswertung in der Grounded Theory ist das »axiale Kodieren«. Hier wird ein handlungstheoretisches Modell auf die im "offenen Kodieren« herausgearbeiteten Kodes gelegt. Juliet M. Corbin und Anselm L. Strauss beschreiben das "axiale Kodieren« als »[e]ine Reihe von Verfahren, mit denen durch das Erstellen von Verbindungen zwischen Kategorien die Daten nach dem offenen Kodieren auf neue Art zusammengesetzt werden. Dies wird durch den Einsatz eines Kodier-Paradigmas erreicht, das aus Bedingungen, Kontext und Handlungs- und Interaktionsstrategien und Konsequenzen besteht. ${ }^{40}$ Im Gegensatz zum handlungstheoretischen Kodier-Paradigma von Corbin und Strauss (Bedingungen, Kontext, Handlungs- und Interaktionsstrategien und Konsequenzen) dient dieser Analyse das handlungstheoretische Modell der Bedingungs-Bedeutungs-Begründungs-Kategorien der Kritischen Psychologie als analytisch heuristischer Rahmen, nach dem die »offenen Kodes« neu zusammengefügt und angeordnet werden. Hier wurde zunächst analog zu den Themen, die sich im offenen Kodieren hervorgetan haben, themenbezogen vorgegangen.

Der letzte Auswertungsschritt der Grounded Theory ist das »selektive Kodieren«. Das »selektive Kodieren« ist der Prozess, in dem auf einem etwas höheren Abstraktionsniveau Kernkategorien ausgewählt werden. Strauss und Corbin verstehen unter Kernkategorie »das zentrale Phänomen, um das herum alle anderen Kategorien integriert sind ${ }^{41}{ }^{41}$ Nachdem nur mit sechs Personen Interviews durchgeführt wurden, konnte dieser Auswertungsschritt nicht in der von Corbin und Strauss und auch von Witzel gedachten Ausführlichkeit durchgeführt werden. ${ }^{42}$ Es konnten allerdings affektive Gemeinschaftsbezüge in ihrer sehr unterschiedlichen Art und Weise als zentrale Kategorien herausgearbeitet werden, die aus subjektiver Perspektive den Eintritt in eine Bewegung mitbestimmen.

40 Anselm L. Strauss-Juliet M. Corbin: Grounded Theory. Crundlagen qualitativer Sozialforschung, Weinheim: Beltz Verlag 1996, S. 75.

41 Ebd., S. 94.

42 Prozesse der Validierung am weiteren Material konnten beispielsweise nicht stattfinden, da alle Interviews bereits sehr ausführlich in die Analysen eingegangen sind. 